\title{
THE ATLAS FORWARD DETECTORS AND THEIR PHYSICS POTENTIAL
}

Benedetto Giacobbe on behalf of the ATLAS collaboration 


\section{outline}

- no $\mathfrak{L}$ uminosity, less physics ...

- the ATLAS Forward Detectors

- luminosity measurements

- physics with FD: $\sigma_{\text {tot }}$

- FD upgrades

- extended physics program with FD

- summary 


\section{relative and absolute luminosity}

- Absolute luminosity:

- measure cross sections for standard physics

- measure Higgs production cross section

- observe deviations from SM and New Physics

- Requirements:

- ultimate precision at the 2-3\% level

- different methods needed for cross check

- minimize systematics

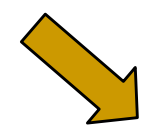

(1). $\mathrm{OH} \rightarrow \gamma \gamma$

四, $\mathrm{tt} H(\mathrm{H} \rightarrow \mathrm{bb})$

$\Delta, \triangle H \rightarrow Z^{(*)} \rightarrow 41$

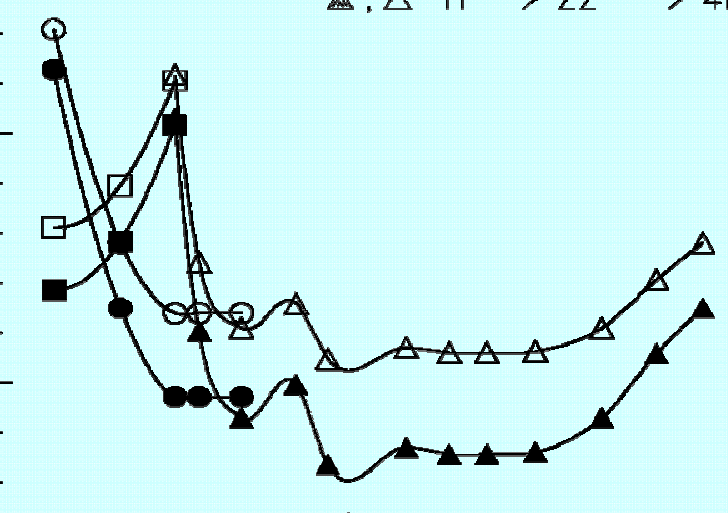

Open symbols : $\Delta L / L=10 \%$

Closed symbols : $\Delta L / L=5 \%$

$10^{2}$

COMBINED EFFORT FROM DIFFERENT DETECTORS, TRIGGER, DAQ..

- Relative luminosity:

- beam stability

- beam degradation (efficient use of trigger)

- evaluate trigger \& DAQ dead-times

- determine beam background

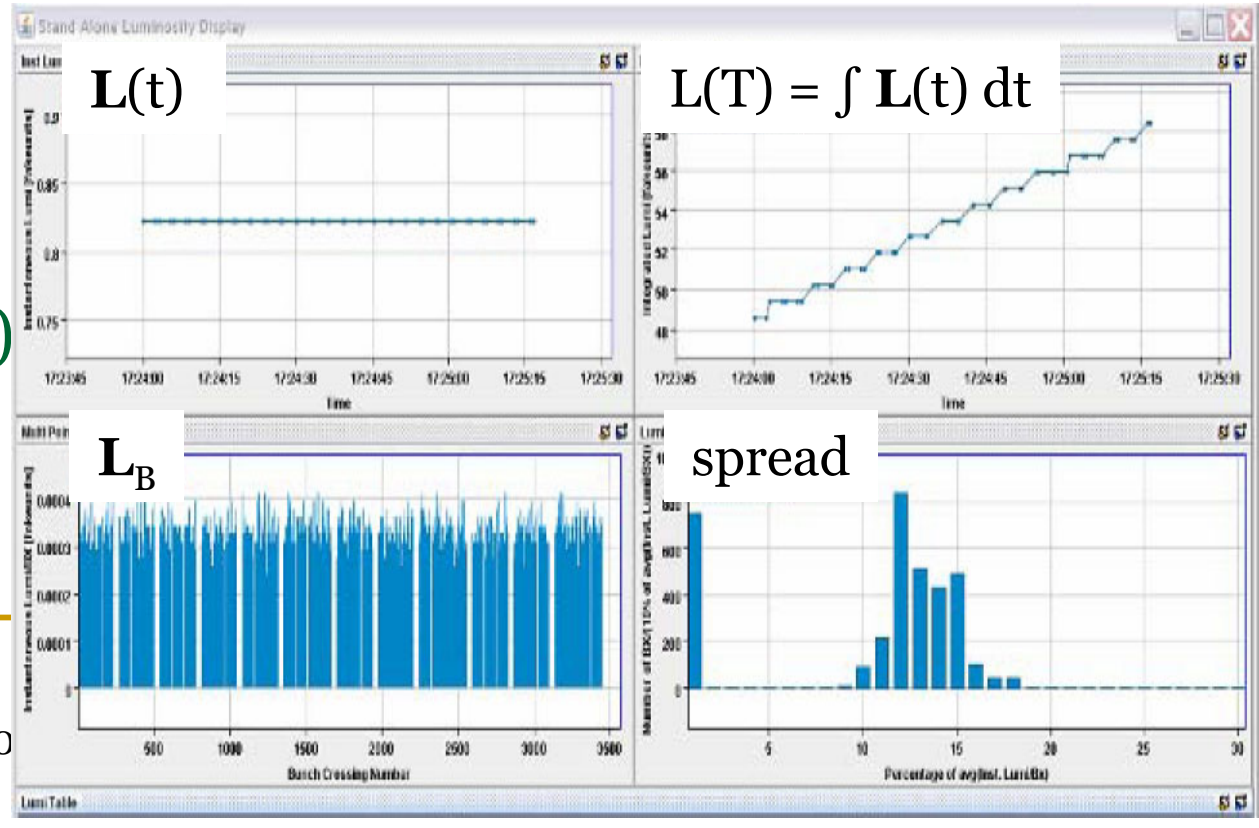




\section{the ATLAS Forward Detectors}

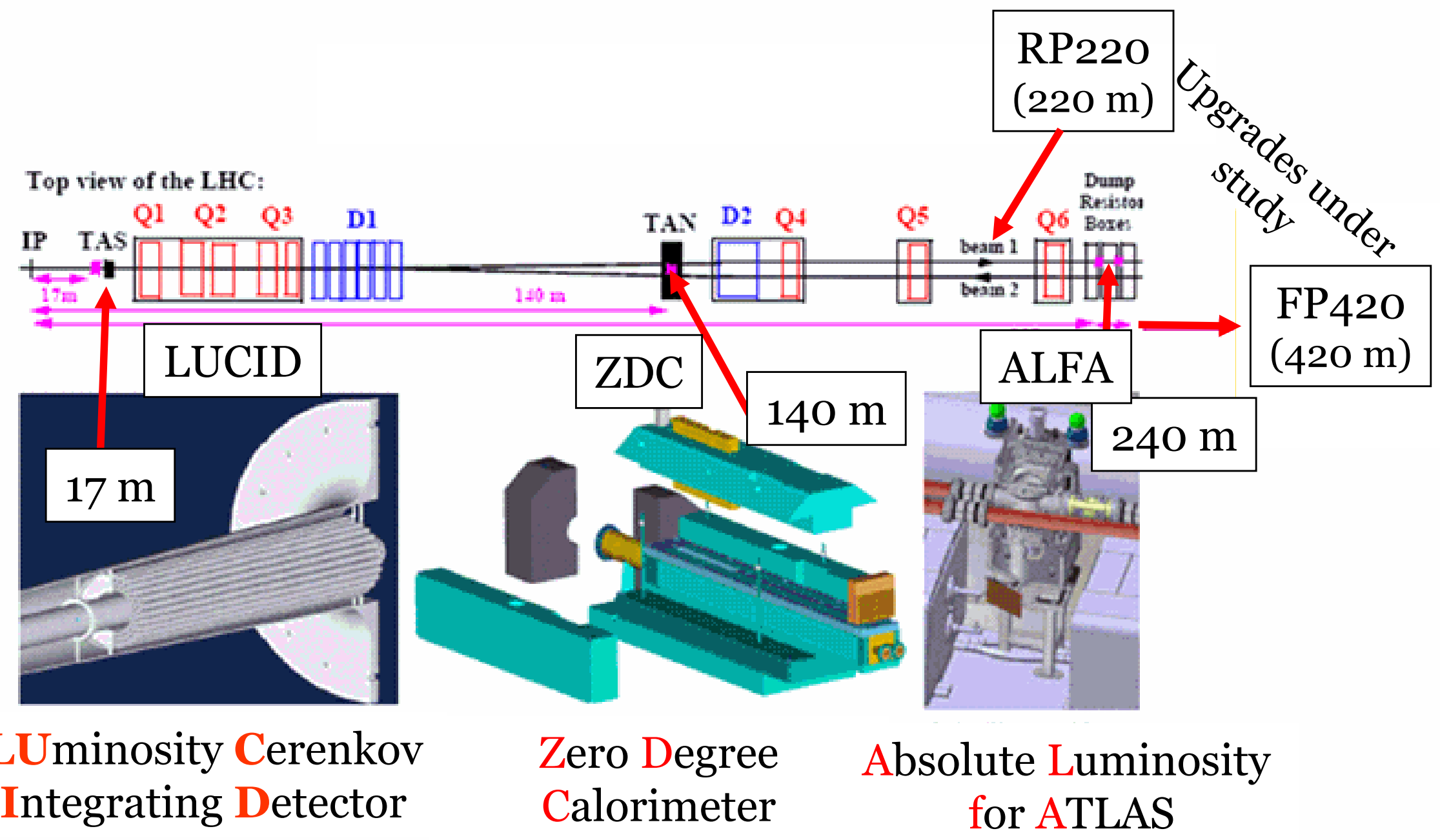




\section{LUCID: where and why}

- Monitor instant. $\mathfrak{~}$

- BC-to-BC structure

- beam degradation

- indep. of LVL1 trigger

- indep. of TDAQ

$\Rightarrow$ Requirements:

- relative $\mathfrak{L}$ sufficient

- fast response (single BC)

- online monitoring

- Measure absolute $\mathfrak{L}$ :

- Needed for phys. analysis

$\Rightarrow$ Requirements:

- calibration needed

- final precision 2-3\%

- Physics capability:

- provide trigger for Forw.Phys. and MB

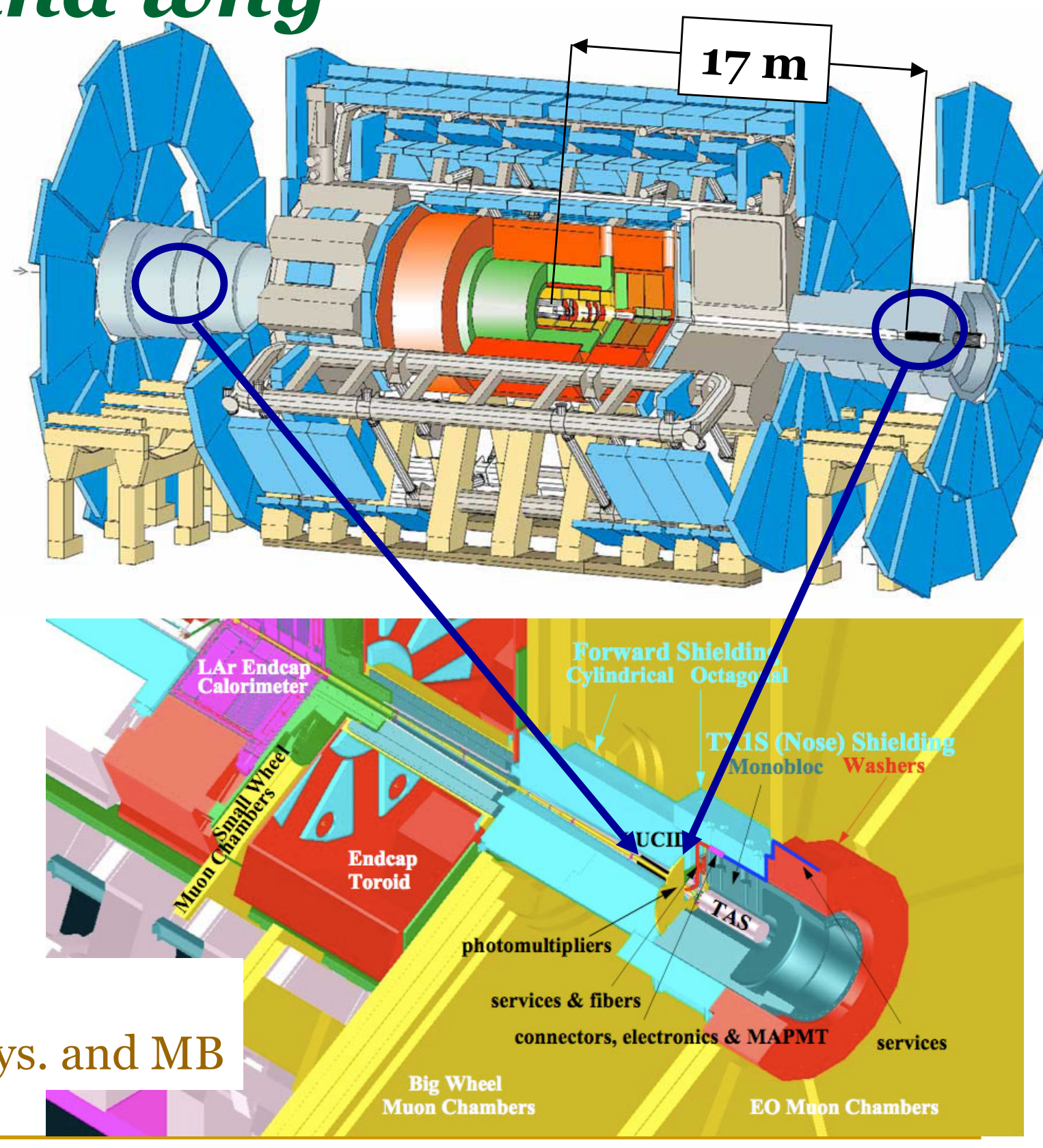




\section{LUCID principle}

Particle from IP

Cerenkov tube

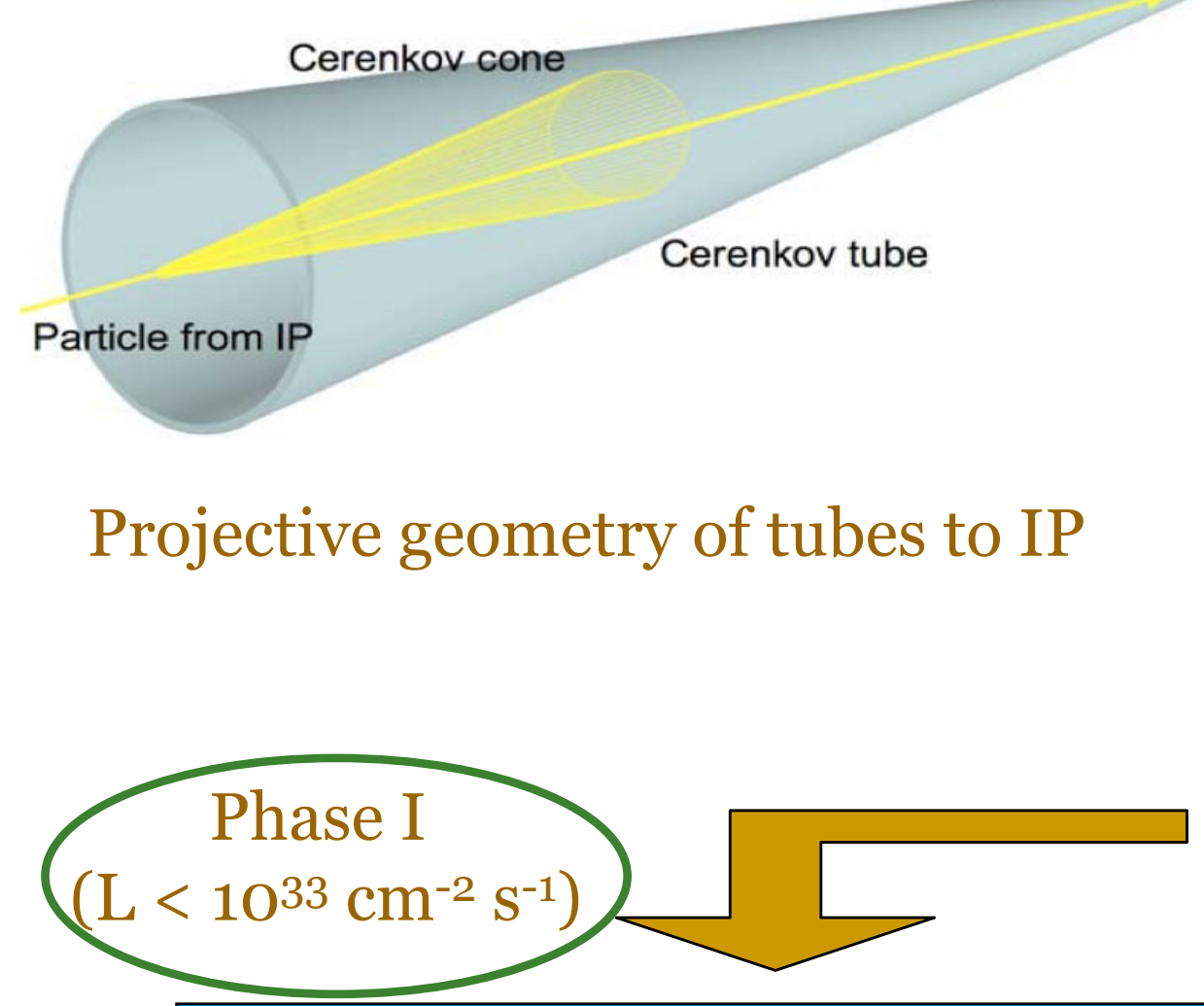

\begin{tabular}{|c|c|}
\hline \multicolumn{2}{|c|}{ Main parameters per module } \\
\hline$\eta$ coverage & $\pm[5.6,6.0]$ \\
\hline N. Tubes & 20 \\
\hline Material & Mechanic. polished Al \\
\hline Gas & C4F10 \\
\hline Pressure & $1.2-1.5$ bar \\
\hline Cherenkov angle & 3 o \\
\hline$<$ N. reflections $>$ & 3 \\
\hline Ch. threshold & $e^{-:} 10 \mathrm{MeV} p: 2.8 \mathrm{GeV}^{2}$ \\
\hline Signal duration & Few $n s$ \\
\hline Read-out & $16 \mathrm{PMTs}+4 \mathrm{fibres}^{-2}$ \\
\hline Expected dose & $7 \mathrm{Mrad} / \mathrm{y} @ 10^{34} \mathrm{~cm}^{-2} \mathrm{~S}^{-1}$ \\
\hline
\end{tabular}

$16 x$

Cherenkov Tube

PMT R762

$4 \mathrm{x}$

Cherenkov Tube

Fibre bundle (PUV700)

MAPMT (H7546B) 


\section{LUCID design for phase I}

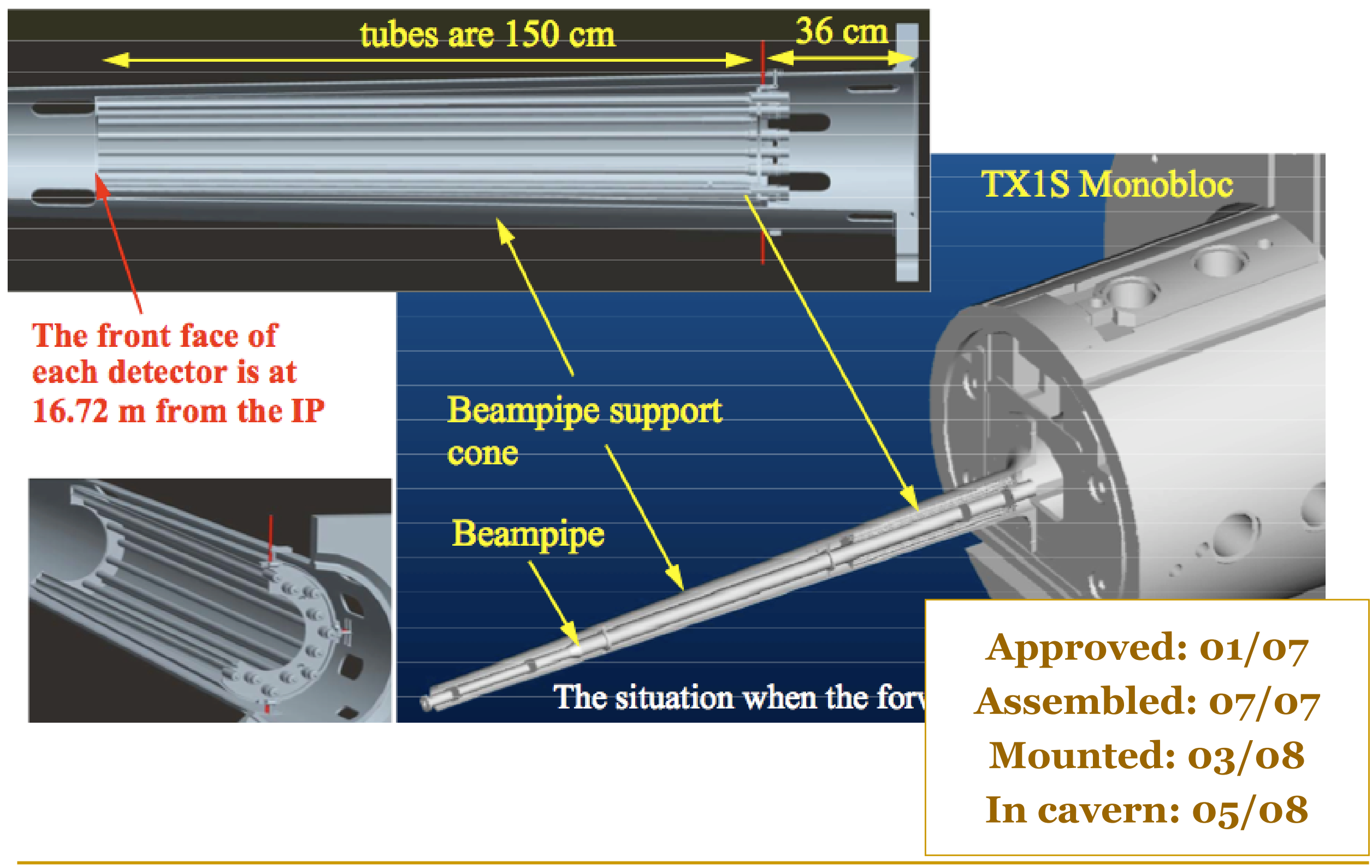




\section{LUCID status}

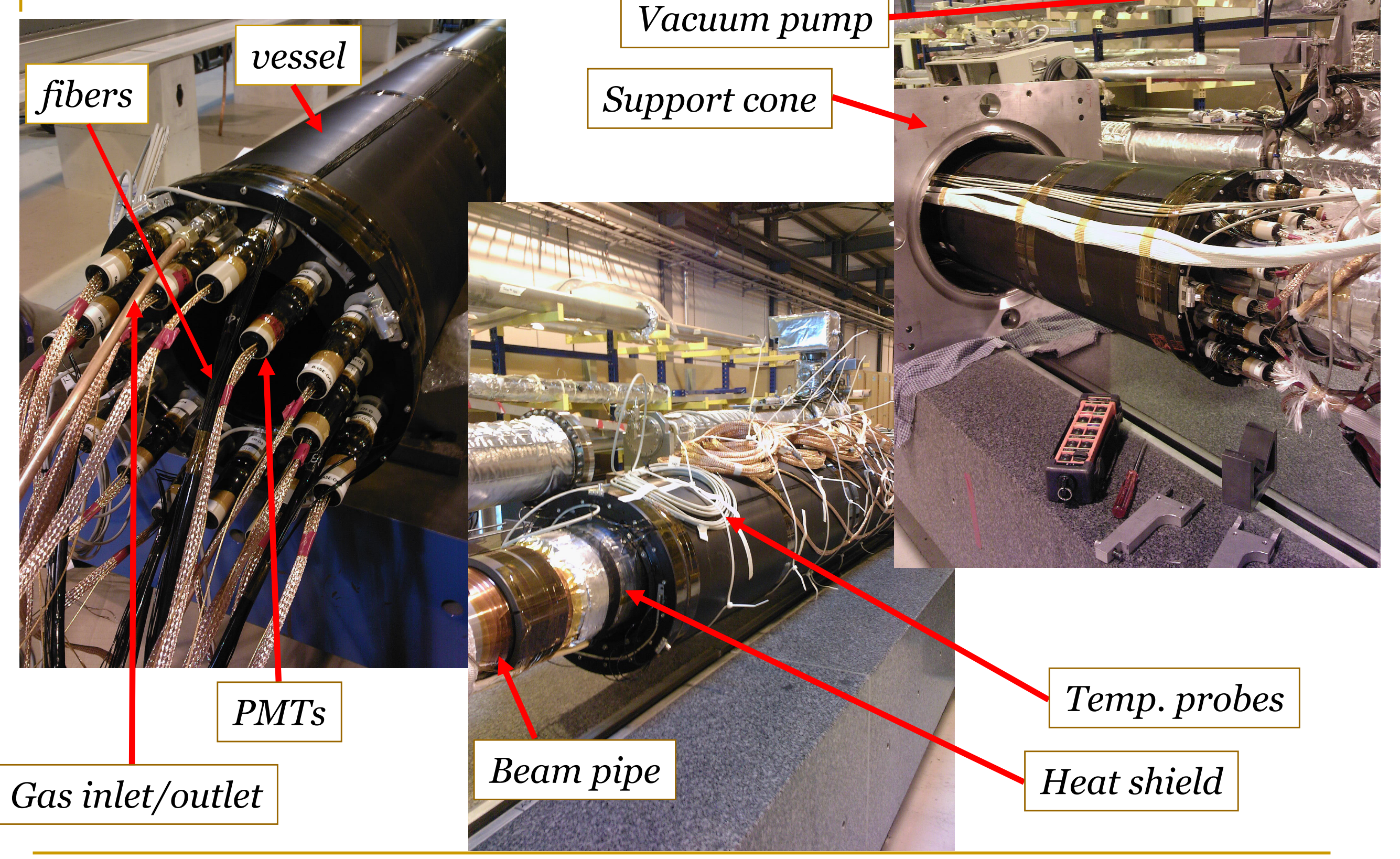




\section{LUCID electronics and trigger}

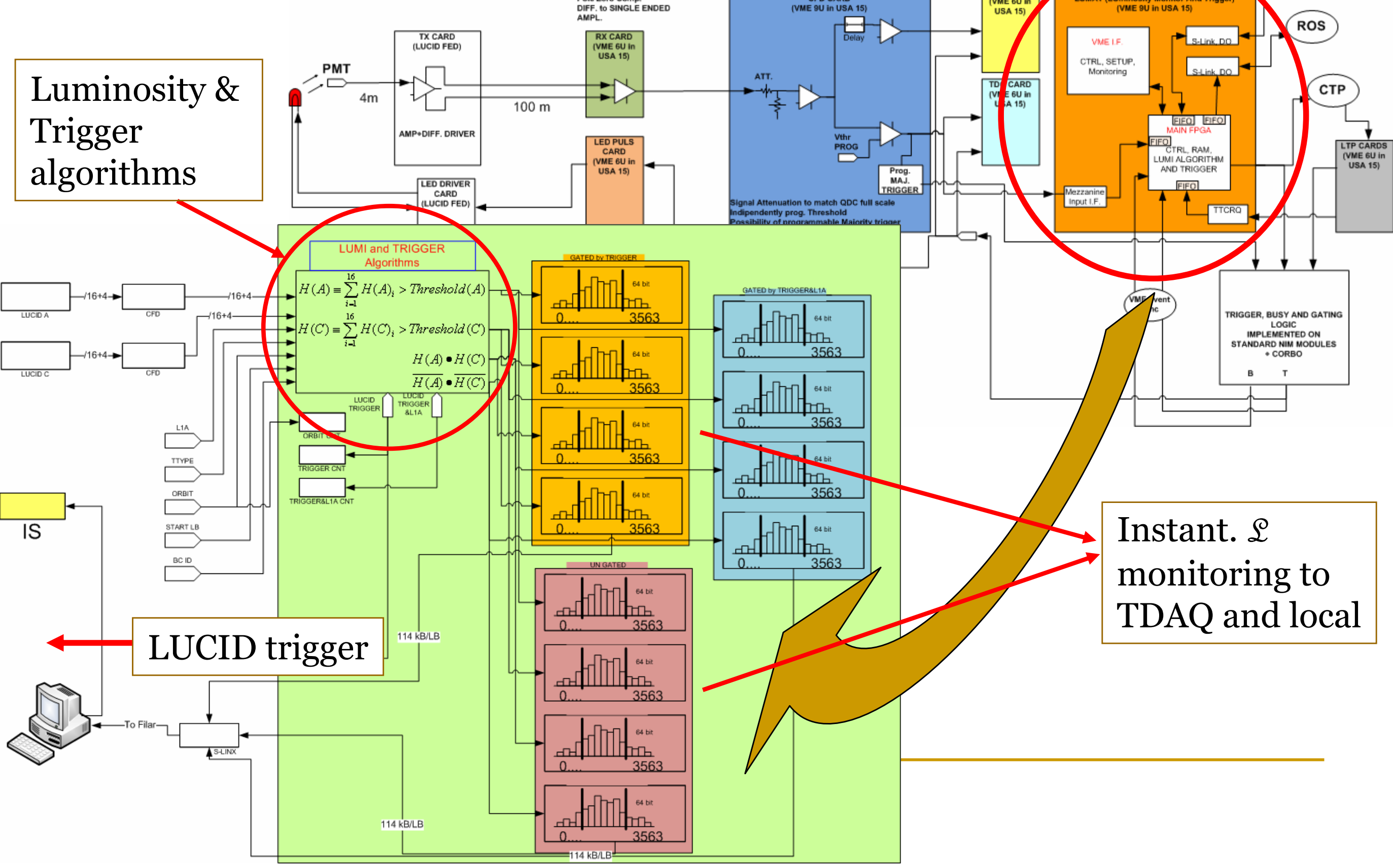




\section{LUCID calibration}

- Relative $\mathfrak{L}$ provided by LUCID from $\mathrm{t}=\mathrm{O}$

- monitor beam stability and structure

口 allow fast reaction to LHC in case of problems

- Absolute $\mathfrak{e}$ needs calibration:

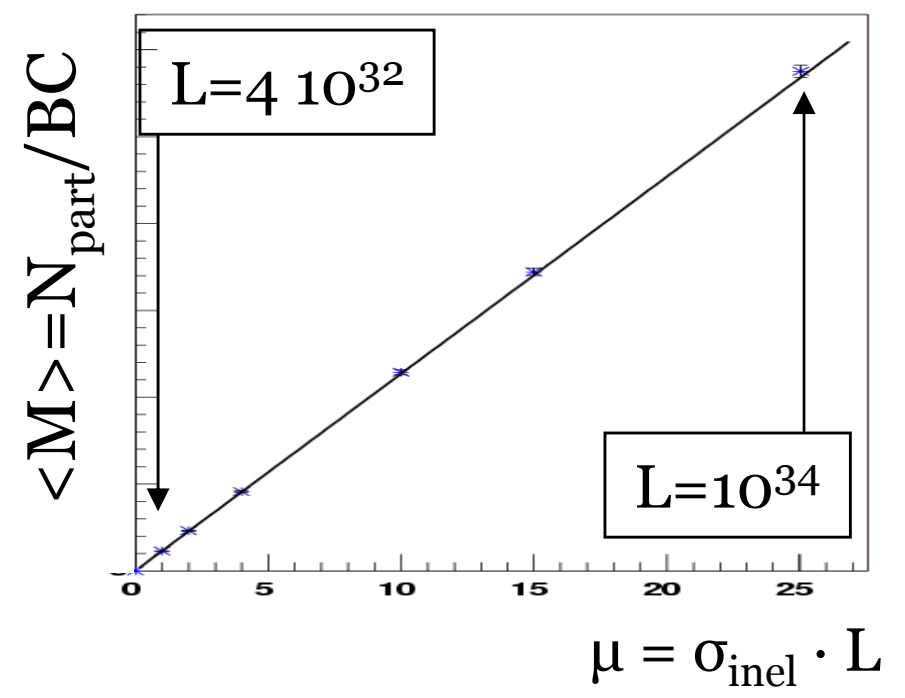

- Different calibration methods foreseen

- Beam parameters ( 10\% precision)

- Physics channels (W, Z) (5-10\% precision)

- ALFA detector (from 2010) (2-3\% precision)

calibration constant:

$\varepsilon_{\mathrm{pp}} \times \sigma_{\text {inel }}$

No need to measure

$\varepsilon_{\mathrm{pp}}$ and $\sigma_{\text {inel }}$ separately!

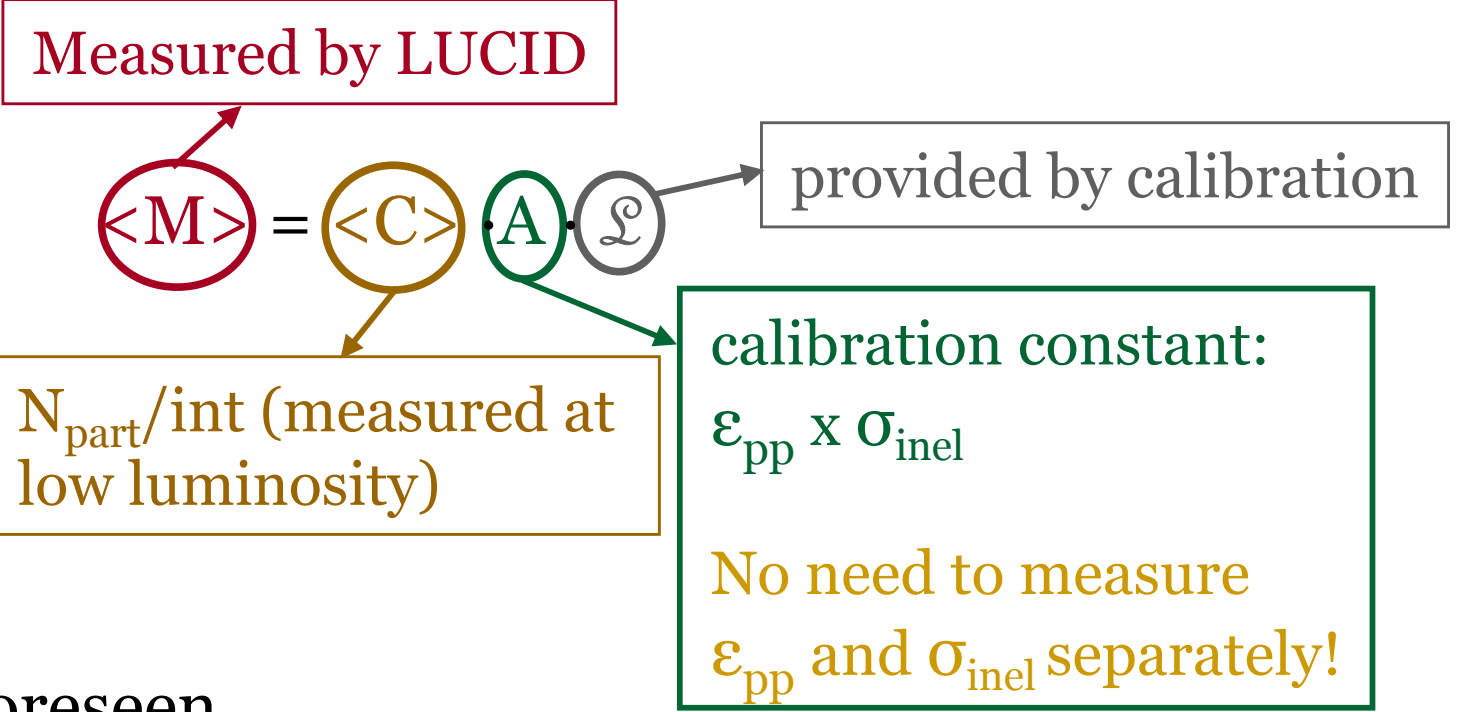




\section{LUCID: absolute $\mathfrak{d}$ determination}

- Two methods foreseen depending on LHC operation:

- only@ low luminosity: zero counting

- low and high luminosity: hit or particle counting

- zero counting method: $\mathrm{n}_{\mathrm{pp}} / \mathrm{n}_{\mathrm{BC}}=1-\exp (-\mathrm{A} \cdot \mathfrak{L}), \mathrm{A}=\mathcal{E}_{\mathrm{pp}} \sigma_{\text {inel }}$

- A from calibration

- no need for particle counting capabilities

- $\quad$ inear at low luminosity $\left(\mathrm{n}_{\mathrm{pp}} / \mathrm{n}_{\mathrm{BC}} \sim \mathrm{A} \cdot \mathfrak{L}\right)$ but $:$ not at high luminosity

口 Can be spoiled by pile up

- Particle counting method: $\mathfrak{L}=<\mathrm{M}>/(<\mathrm{C}>\cdot \mathrm{A}), \mathrm{A}=\mathcal{E}_{\mathrm{pp}} \sigma_{\text {inel }}$

- A from calibration

- Need for particle counting capabilities

- Linear relation between $<\mathrm{M}>$ and $\mathfrak{L}$

- Suitable at both low and high luminosity 


\section{ALFA where and why}

- Determine absolute $\mathfrak{L}$ with elastic proton scattering:

- Coulomb normalisation

- Optical theorem

- calibrate LUCID

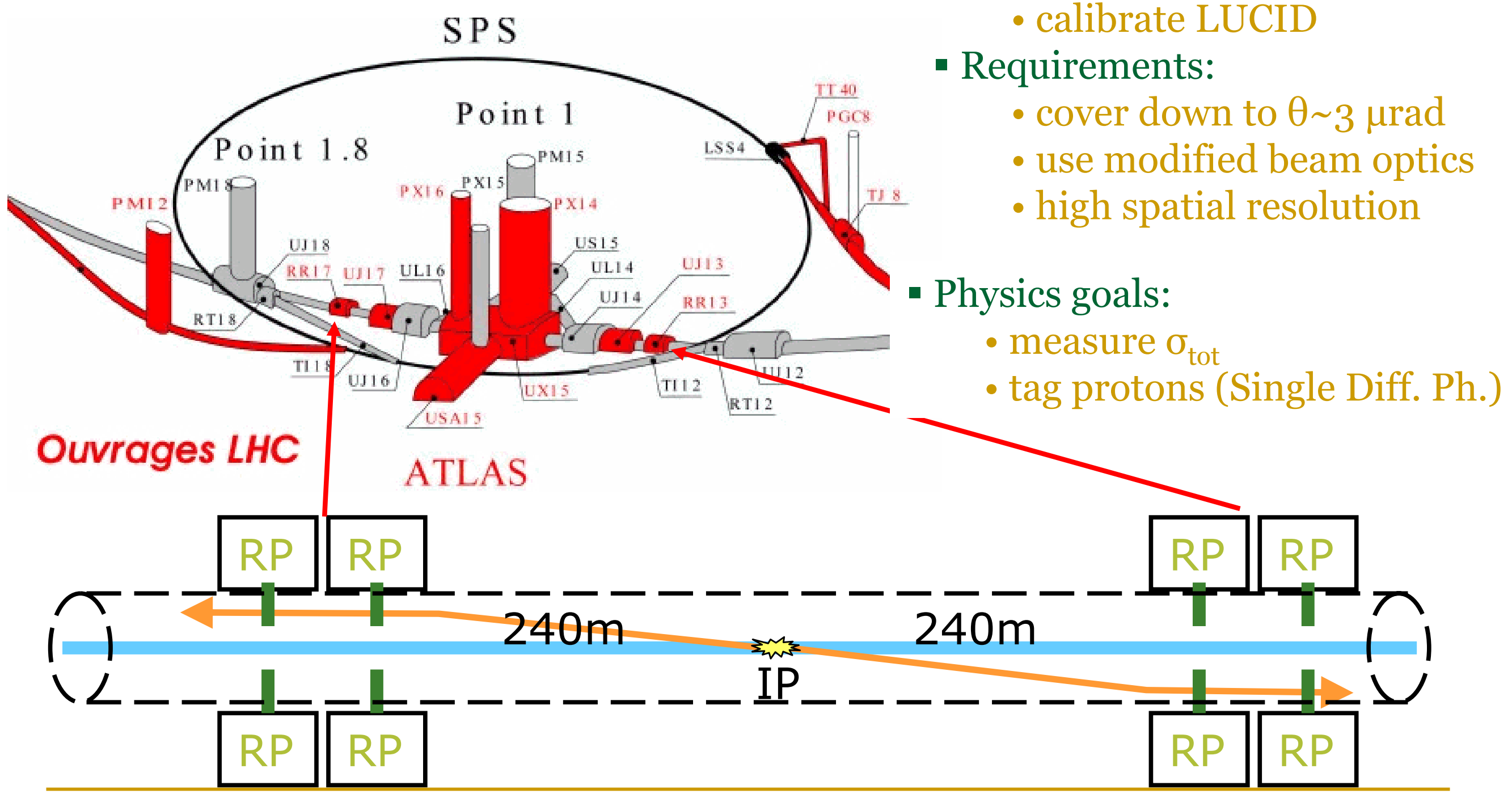




\section{ALFA Roman Pots concept}

\section{Extracted position}

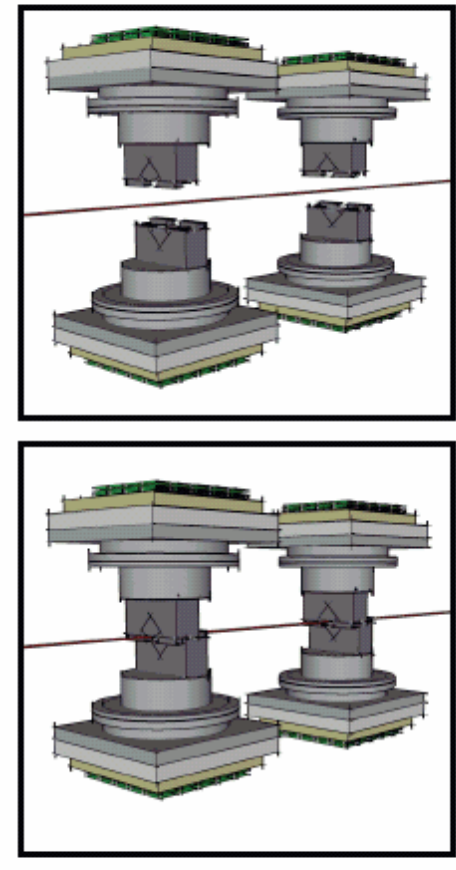

Working position

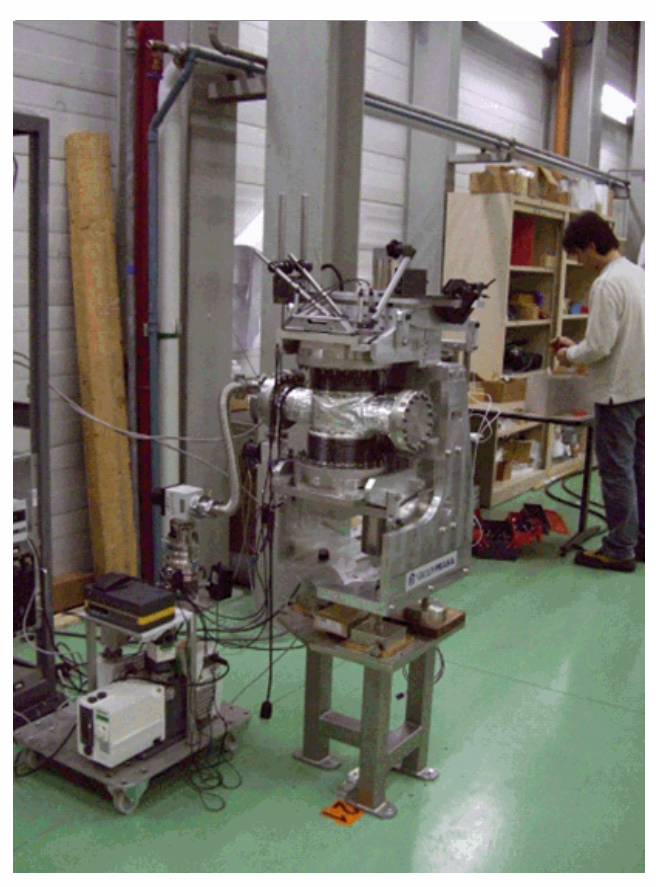

Roman Pot unit

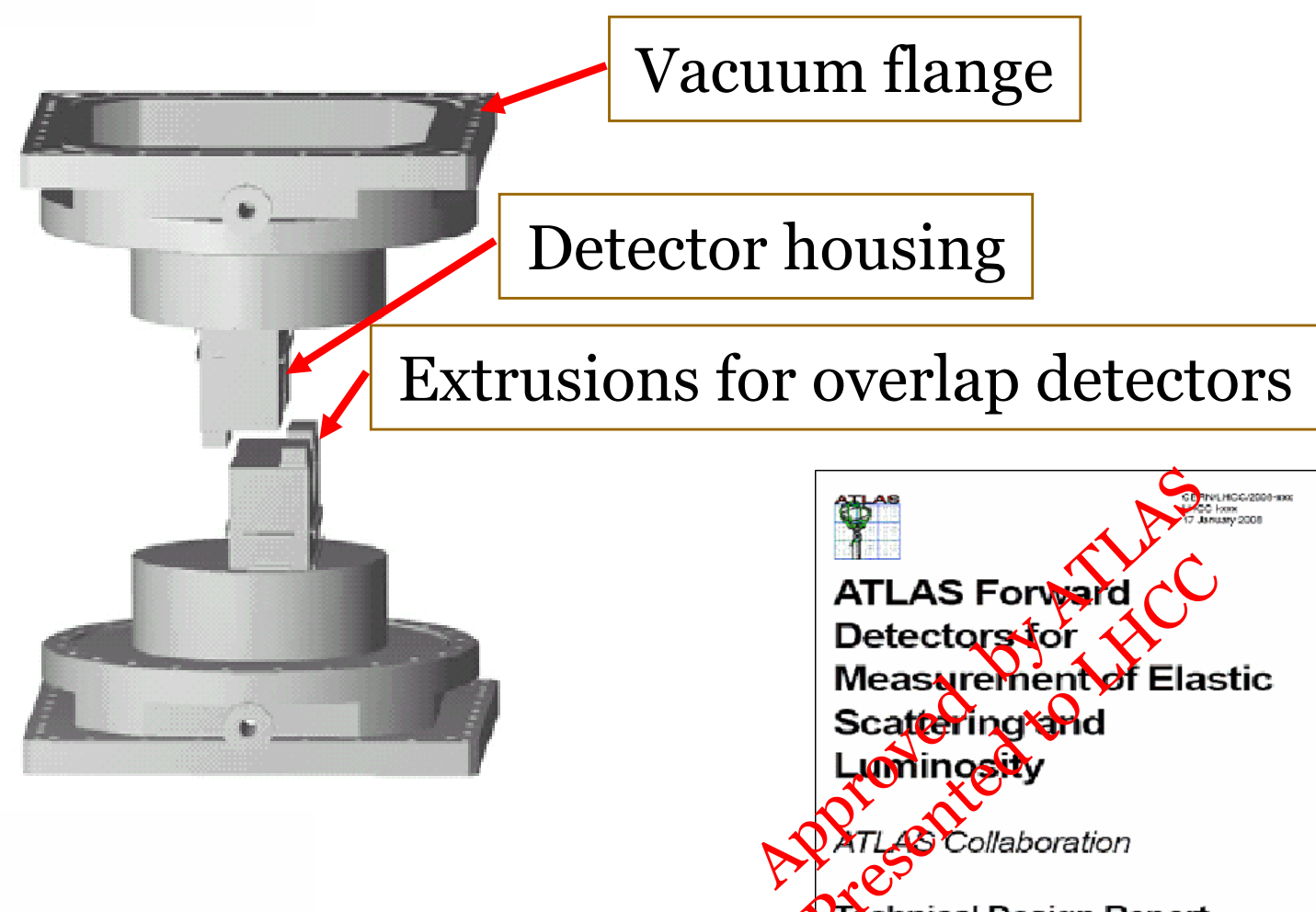

RTechnical Design Report

- Proven techique for approaching very close to beam $(10 \sigma \sim 1 \mathrm{~mm})$

- 2 units $4 \mathrm{~m}$ apart in each side of IP $\Rightarrow 8$ independent pots

- Thin windows to approach active detector close to beam and minimize material

- precise positioning given by Overlap Detectors

\section{STRINGENT LIMITS IMPOSED BY LHC CONSTRAINTS}




\section{ALFA detectors}
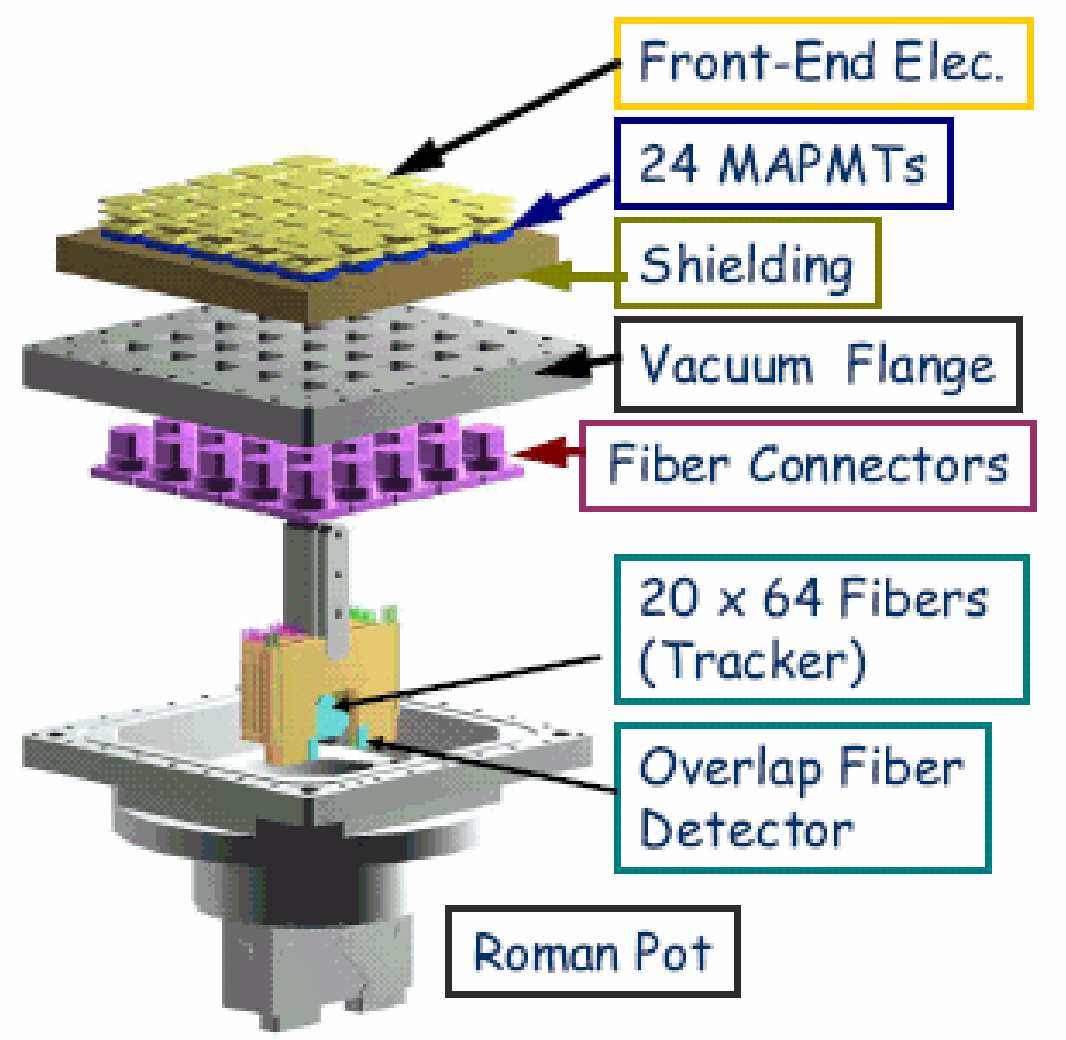

- OD: scintillating fibres

- measure vertical coord. only

- detect beam halo

- each made of 3 planes of 30 fibres
- Requirements:

- measure $|\mathrm{t}| \sim 6 \cdot 10^{-4}(\theta \sim 3 \mu \mathrm{rad})$

- $\sigma_{\mathrm{xy}}<<\sigma_{\text {beam }}(\sim 130 \mu \mathrm{m}) \Rightarrow 30 \mu \mathrm{m}$

- radiation $\leq 100 \mathrm{~Gy} / \mathrm{yr}$

- time resolution $\sim 5 \mathrm{~ns}$

- top/bottom alignm. $\sim 10 \mu \mathrm{m}$

- vacuum tight

- Solution: scintillating fibres detector

- edgeless $(\sim 10 \mu \mathrm{m})$

- 10 x $64 \mathrm{U}+10 \times 64 \mathrm{~V}$ fibres @ 90

- planes staggered by $70.7 \mu \mathrm{m}$

- fibre eff. pitch 50 $\mu \mathrm{m}$ ( $\sigma x y \sim 20 \mu \mathrm{m})$

- MAPMT read out fibres

- FE mounted directly on top of pot

Plastic scintillators cover active fibers area and provide local trigger

Goal: detector ready in $\sim 1$ year from now 


\section{absolute luminosity \& $\sigma_{\text {tot }}$}

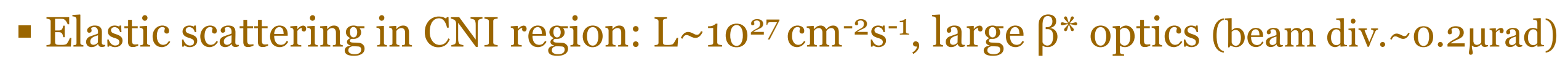

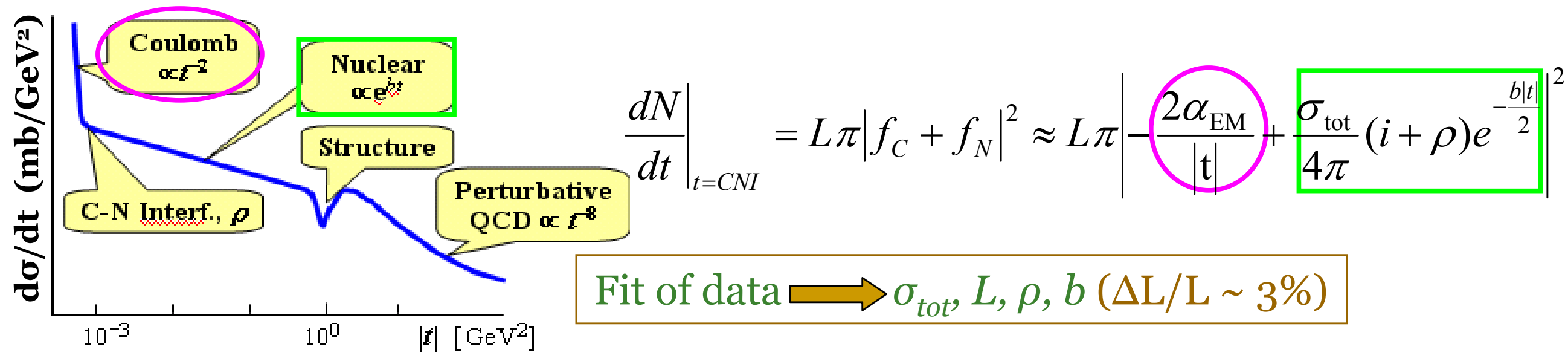

- optical theorem as complementary solution

$$
\left\{\begin{array}{l}
L=\frac{\left(1+\rho^{2}\right)}{16 \pi} \frac{N_{\text {tot }}^{2}}{\left.\frac{d N_{\text {el }}}{d t}\right|_{t=0}} \\
\sigma_{\text {tot }}=\frac{N_{\text {tot }}}{L}
\end{array}\right.
$$$$
\mathrm{N}_{\mathrm{tot}}, d N_{\mathrm{el}} /\left.d t\right|_{t=O} \Rightarrow L \& \sigma_{\text {tot }}
$$$$
\text { : }) \text { need MC for } \eta \text { extrapolation }
$$$$
\oplus \rho \text { shouldn't be a problem }
$$

- Provide high precision (2-3\%) LUCID calibration 


\section{ZDC where and why}

- Requirements:

- measure neutral particles at $\mathrm{O}^{\circ}\left(n, \gamma, \pi^{0}\right)$

- both EM and HAD sections

- beam monitoring and tuning

- crossing angle

- IP position

- lumi monitor at the single BC level

- tune LHC parameters in first times

- radiation hard

- Physics program:

- pp physics: very forward $(\eta>8.3)$ cross sections

- Heavy Ions: event centrality, trigger

LOI presented in January 2007 (CERN-LHC-2007-001)

Status: installed both arms in a simplified version system and electronics ready for first protons 


\section{ZDC}

- 1 EM $\left(29 X_{0}\right)+3$ HAD $\left(1.14 \lambda_{\text {int }}\right)$ modules/arm

- $11 \mathrm{~W}$ plates / module

- $1 \mathrm{~mm}$ quarz rods // to beam (E, x/y meas.) Cherenkov light read out by MAPMT

- $1.5 \mathrm{~mm}$ quarz strips (E meas.) $\perp$ to beam read out by $\mathrm{PM}$

- in HAD modules RO simplified measured quarz radiation hardness:

- small degradation up to $5 \mathrm{Grad}$

- at $10^{33}$ ok for few years

- at $10^{34}$ will survive few months
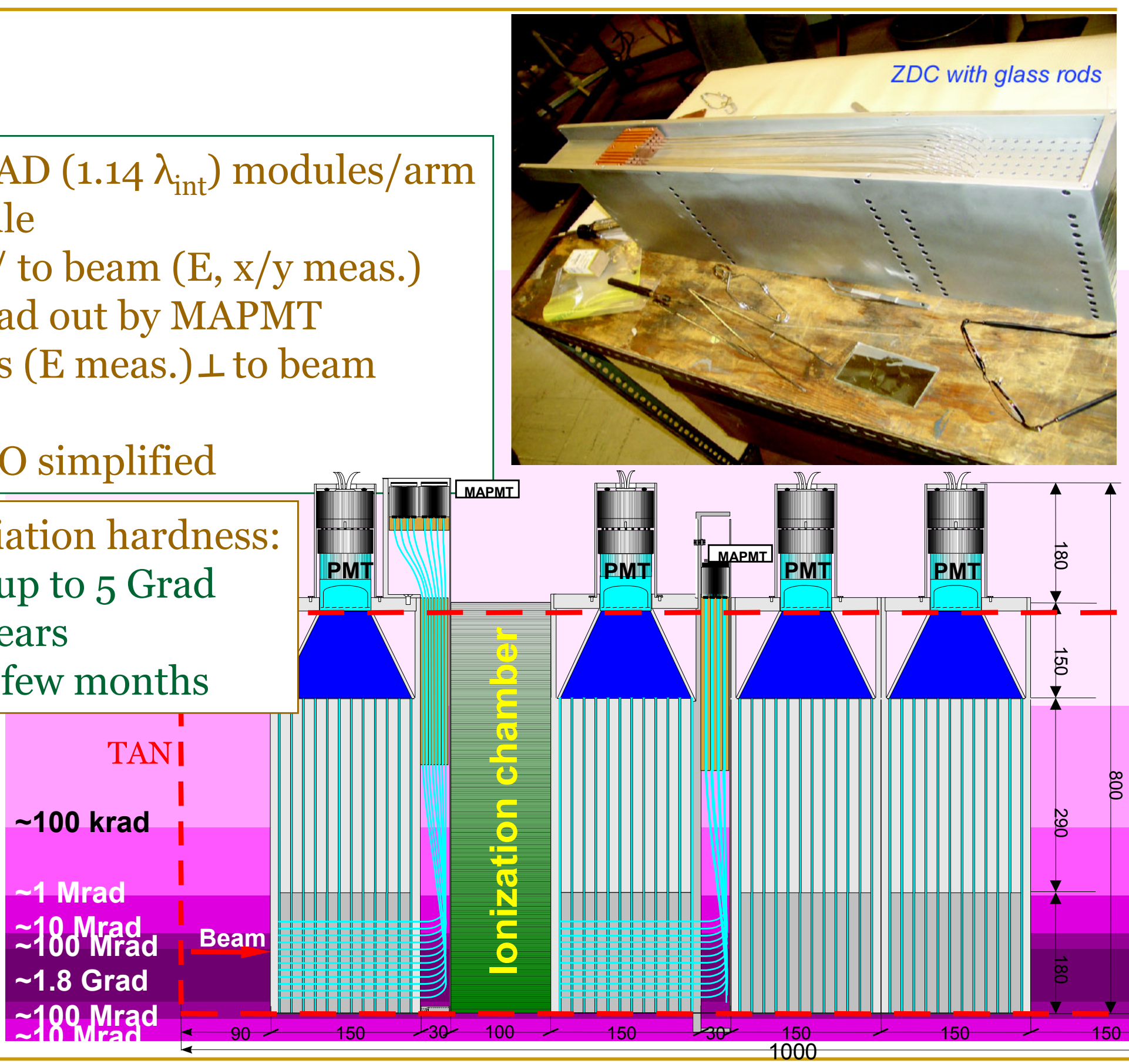

PMT
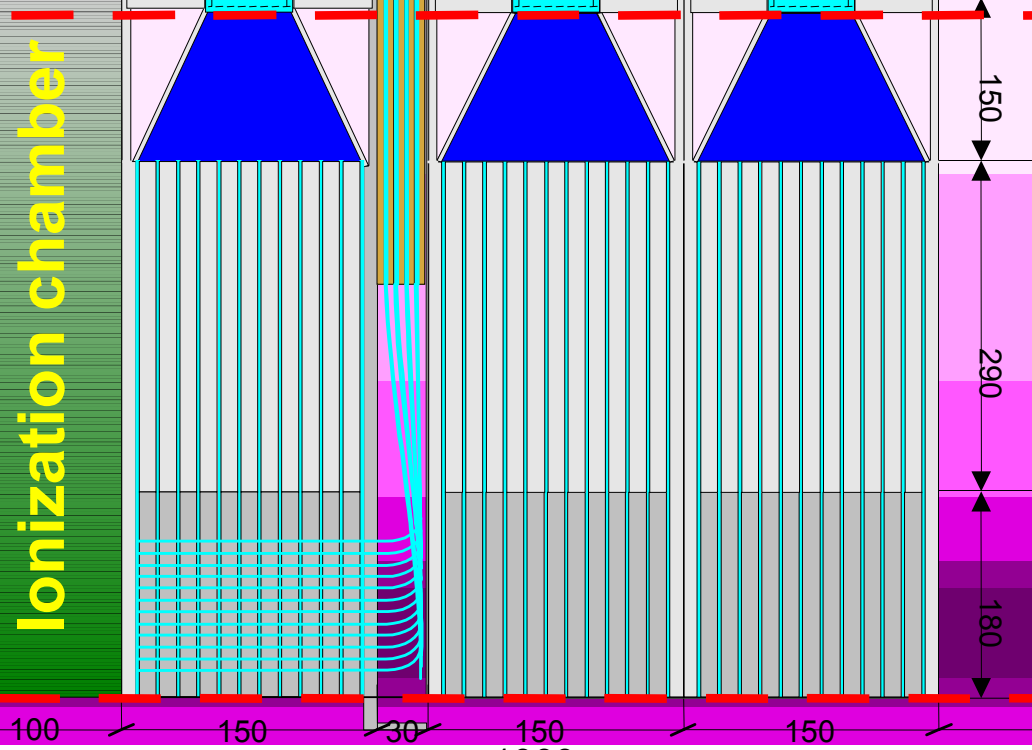


\section{physics with ZDC: pp \& heavy ions}

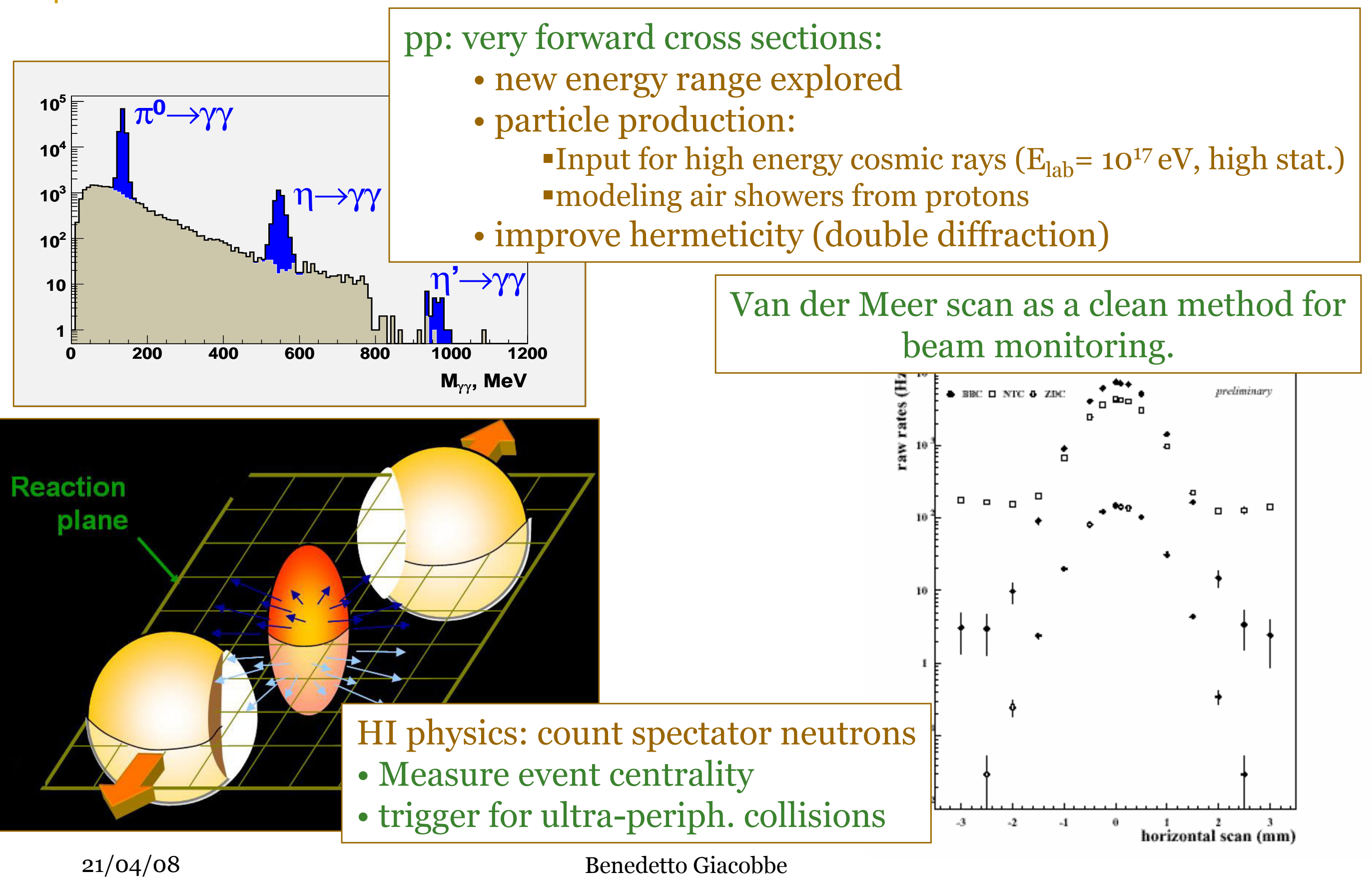




\section{RP22O \& FP42O upgrades}

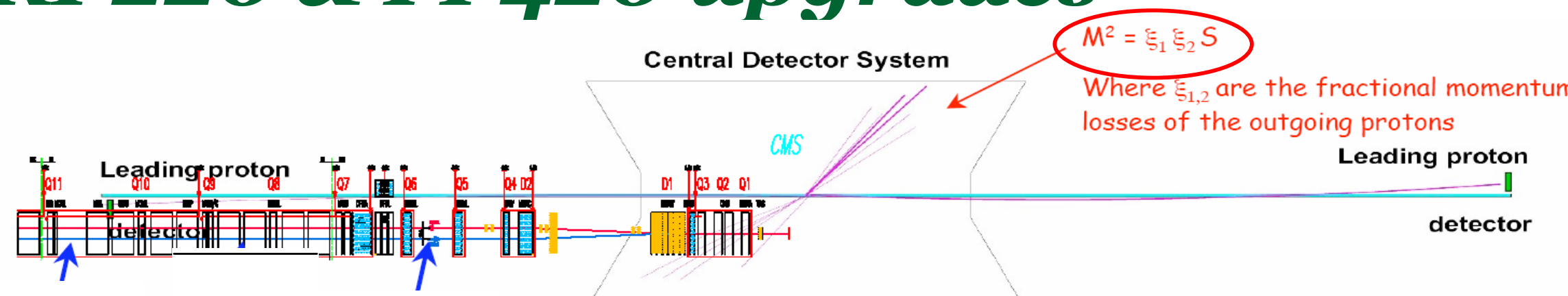

$\mathrm{RP} 420$

RP220

- At nominal LHC running conditions:

220m: $0.02<\xi<0.2$

$\sim 420 \mathrm{~m}: 0.002<\xi<0.02 \quad(\mathrm{M}>\sim 30 \mathrm{GeV})$

- Requirements:

- Close to the beam $=>$ edgeless detectors

- High lumi operation $=>$ radiation hard

- Few $\mu \mathrm{m}-1 \mu \mathrm{rad}$ precision

- Suppress pile-up => add fast timing det.

- Status of projects:

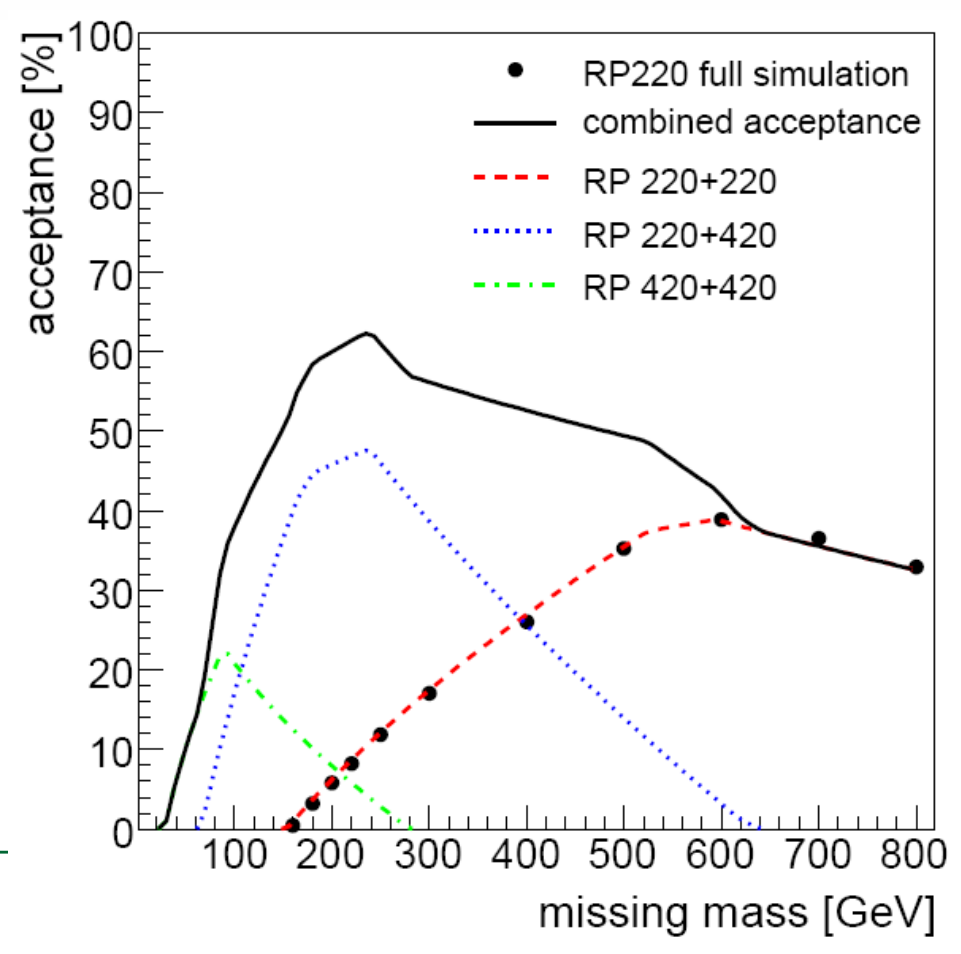

- R\&D advanced: conclusions ready and submitted by end 2008 for final decision

- test beam campaign organised 


\section{diffractive physics}

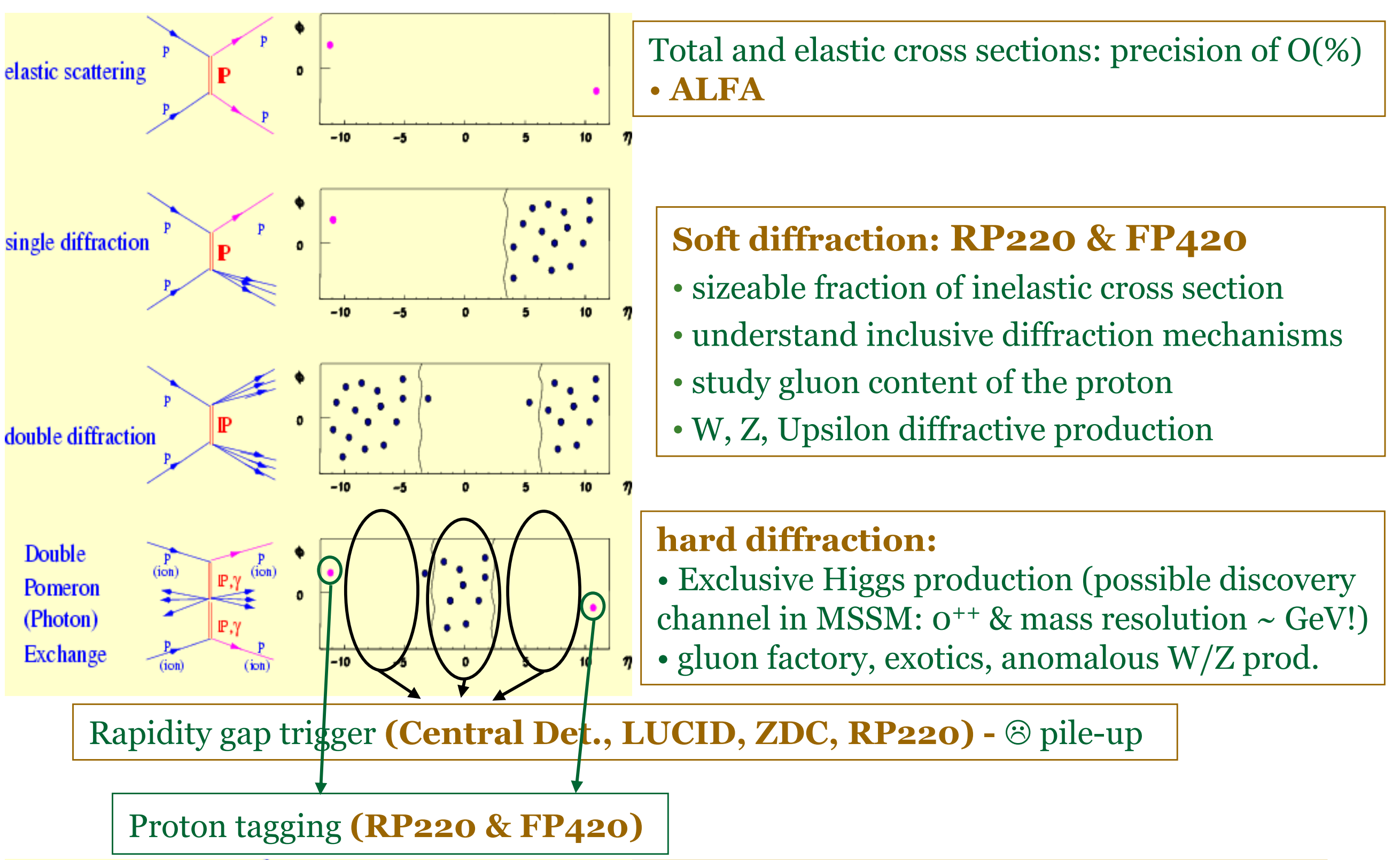




\section{conclusions}

- ATLAS Forward Detectors will allow $\mathfrak{L}$ determination at 2$3 \%$ level, monitor beam conditions down to the BC scale and measure $\sigma_{t o t}$

aUCID and ZDC installed and ready for beam

$\checkmark$ ALFA ready in 1 year

- RP220-FP420 upgrade of ATLAS under study

a $\quad$ R\&D advanced. Conclusions ready and submitted by end 2008 for final decision.

- forward physics program extension foreseen

a wide diffractive physics program possible

a can prove to be a key field for new physics 


\section{Backup slides}




\section{ZDC time, space and energy resolution (average over active area)}
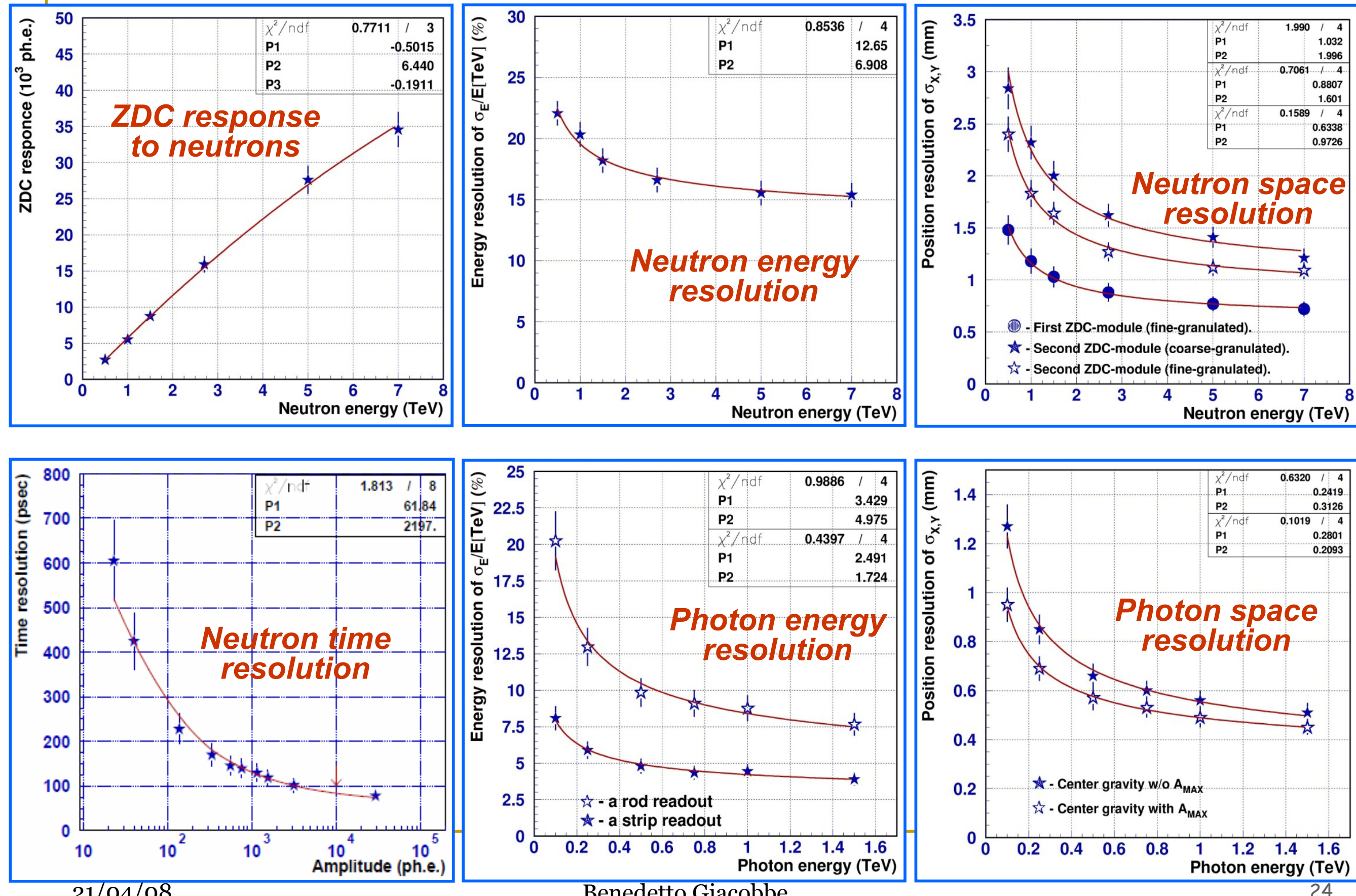


\section{ATLAS Detectors for Luminosity Determination and Forward Physics}

Baseline ATLAS detector covers $-5<\eta<5$

Tracking $-2.5<\eta<2.5$

However $\eta_{\max } \sim \ln \mathrm{s} / \mathrm{m}_{\mathrm{p}} \sim 9.5$

Large region in rapidity not covered

(Angles from o.8 degree to o degrees)
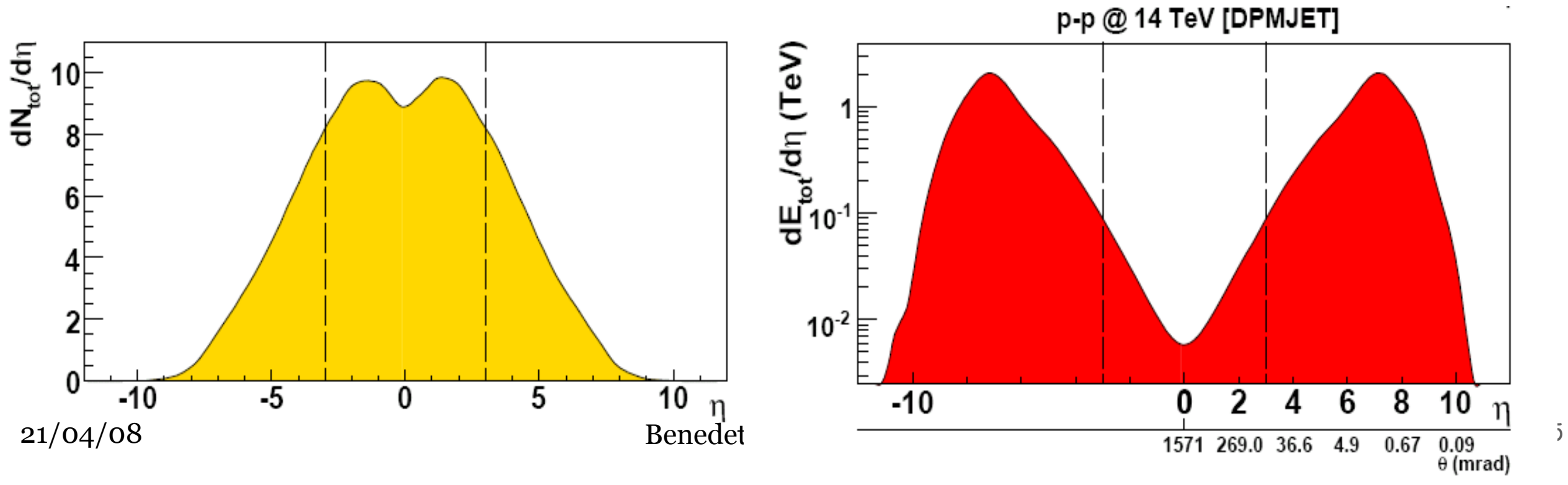


\section{Trigger conditions}

- For the special run $(\sim 100 \mathrm{hrs}, \mathrm{L}=1027 \mathrm{~cm}-2 \mathrm{~s}-1)$

- 1. ALFA trigger

- coincidence signal left-right arm (elastic trigger)

- each arm must have a coincidence between 2 stations

- rate about $30 \mathrm{~Hz}$

- 2. LUCID trigger

- coincidence left-right arm (luminosity monitoring)

a single arm signal: one track in one tube

- 3. ZDC trigger

a single arm signal: energy deposit $>1 \mathrm{TeV}$ (neutrons)

- 4. Single diffraction trigger

- ALFA.AND.(LUCID.OR.ZDC)

- central ATLAS detector not considered for now (MBTS good candidate) 


\section{Single diffraction: trigger conditions}

\begin{tabular}{|c|c|c|}
\hline Efficiency [\%] & Pythia & Phojet \\
\hline Preselection & 97.1 & 94.8 \\
\hline ZDC [E>1 TeV] & 51.5 & 38.7 \\
\hline LUCID [1 track] & 45.1 & 57.3 \\
\hline $\begin{array}{c}\text { [Central ATLAS E }>\text { 100 } \\
\text { GeV] }\end{array}$ & 24.9 & 38.7 \\
\hline Total preselection & 75 & 74 \\
\hline RP selection & 60.1 & 54.2 \\
\hline $\begin{array}{c}\text { ALFA } \\
\text { (Relative to preselection) }\end{array}$ & 44.9 & 40.1 \\
\hline Total acceptance & & \\
\hline
\end{tabular}




\section{Exclusive Higgs production}

\section{Generator studies with detector cuts}

Standard Model Higgs

b jets : $\quad M_{H}=120 \mathrm{GeV} \mathrm{s}=2 \mathrm{fb}$ (uncertainty factor $\sim 2.5$ )

$$
\mathrm{M}_{\mathrm{H}}=140 \mathrm{GeV} \mathrm{s}=0.7 \mathrm{fb}
$$

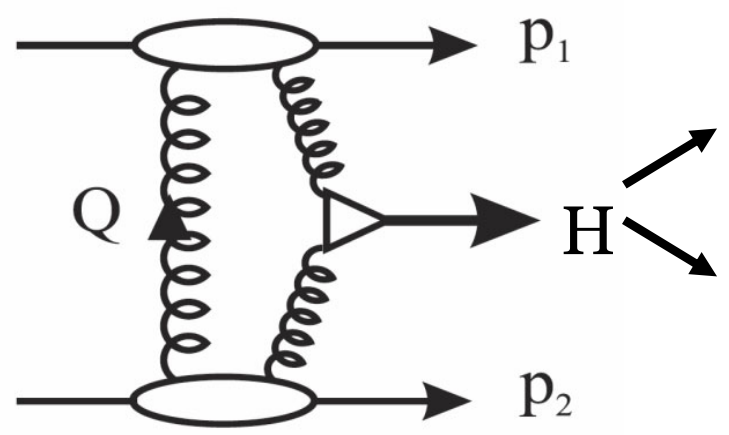

$$
\begin{array}{cc}
\mathrm{M}_{\mathrm{H}}=120 \mathrm{GeV}: 11 \text { signal } / \mathrm{O}(10) \text { background in } 30 \mathrm{fb}^{-1} \\
\text { hep-ph/o207042 }
\end{array}
$$

$$
\begin{aligned}
& W^{*}: \quad M_{H}=120 \mathrm{GeV} \mathrm{s}=0.4 \mathrm{fb} \\
& \mathrm{M}_{\mathrm{H}}=140 \mathrm{GeV} \mathrm{s}=1 \mathrm{fb} \\
& \mathrm{M}_{\mathrm{H}}=140 \mathrm{GeV}: 8 \text { signal / O(3) background in } 30 \mathrm{fb}^{-1} \\
& \text { hep-ph/0505240 } \\
& \text { with detector cuts }
\end{aligned}
$$

- The $b$ jet channel is possible, with a good understanding of detectors and clever level 1 trigger (need trigger from the central detector at Level-1, possibly with $\mathrm{O}(10) \mathrm{KHz}$ rate)

-The $\mathrm{WW}^{*}(\mathrm{ZZ})$ channel is extremely promising : no trigger problems, better mass resolution at higher masses (even in leptonic / semi-leptonic channel) 


\section{Higgs Studies}

Central exclusive production

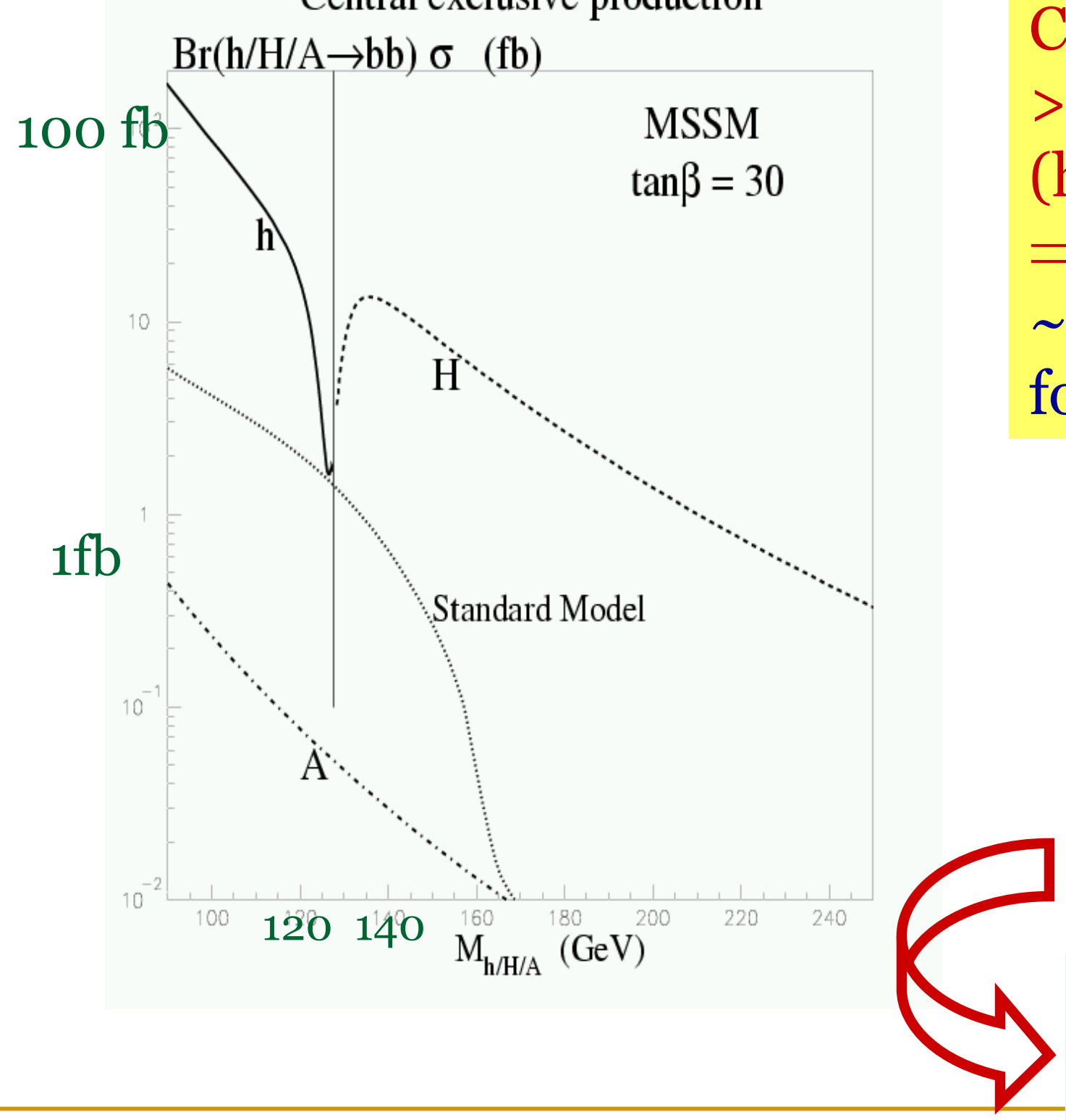

Cross section factor

$>10$ larger in MSSM

(high $\tan \beta$ )

$\Rightarrow$ Few 100 events with

$\sim 10$ background events for $30 \mathrm{fb}^{-1}$

Kaidalov et al., hep-ph/0307064

$\Rightarrow$ Study correlations between the outgoing protons to analyse the spin-parity structure of the produced boson

A way to get information on the spin of the Higgs $\Rightarrow$ ADDED VALUE TO LHC 